\title{
Does the Short Period of Resistance Training May Improve the Sleep Quality? Pilot Study
}

\author{
Luis Felipe Tubagi Polito ${ }^{1,2,3,6}$, Douglas Popp Marin',4, Carla Giuliano Pinto de Sá Montenegro ${ }^{5}$, \\ Marcelo Callegari Zanetti6,7, Marcelo Villas Boas Junior ${ }^{3,6,7}$, Helton Magalhães Dias ${ }^{3,6}$, Roberta Luksevicius Rica ${ }^{6}$, \\ Júlia Mascarenhas', Aylton Figueira Junior6, Maria Regina Ferreira Brandão ${ }^{6}$
}

\begin{abstract}
Purpose: The present study aimed evaluated the influence of exercise program on sleep quality of sedentary individuals. Methods: The study evaluated 30 subjects after resistance training session constituted by six multi-joints exercises (leg press, chest press, machine let pull down, seated machine shoulder press, crunches and machine trunk extension) three times a week for 40 days. The intensity of the effort was controlled using the follow criteria: the ideal repetitions for each set was between eight and fifteen. The Pittsburgh Sleep Quality Questionnaire (PSQI) was applied before and after training period. Results: There was a significant effect of short-term resistance training on all sub-scores of PSQI $(p<0.05)$, except for sleep duration $(p>0.05)$. Conclusion: The resistance training program was able to improve the sleep quality in healthy subjects. In addition, the applicability of low-cost instruments (psychometric instruments like PSQI) to evaluate health parameters, which could be strengthened by other studies.
\end{abstract}

Key words: Resistance Training; Strength; Physical Exercise; Sleep.

\section{INTRODUCTION}

Circadian rhythms determine rhythmic changes in physiological and behavioral aspects, such as body temperature, cortisol levels, melatonin and growth hormone, mood, cognitive ability, and especially the sleep and wake cycle. Indeed, being awake or sleeping at different times of the day and night is not only due to a situation of fatigue or exhaustion, but rather due to the innate rhythms that determine the pattern of days and nights ${ }^{(1)}$.In general, the regulation and control of the sleep and wake cycle are attributed to the hypothalamic systems and their respective functional interactions with other structures of the central nervous system, in particular, the cerebral cortex ${ }^{(2,3)}$. In this sense, sleep is a fundamental and cyclical process of the organism, which exerts several biological and psychological functions, for example, the energy metabolism restoration and the memory consolidation; which can be conceptualized as a reversible behavioral state, in which characteristic features such as relaxed posture, minimal movements, perceptive disengagement and increased threshold of responses to external stimuli can be observed ${ }^{(4-7)}$. Additionally, sleep consists of alternating stages that are differentiated by their EEG pattern and are classified as: NREM sleep (Non-Rapid Eye Movements) and REM sleep (Rapid Eye Movements). The NREM stage is characterized by greater stability, sleep depth or slow wave sleep, as well as the functional prevalence of parasympathetic influences associated with the low activity of sympathetic activity. In contrast, the REM stage is characterized by the great variability in sympathetic activity associated with changes in tonic parasympathetic discharge ${ }^{(4,8)}$ However, psychosocial, pharmacological, and lifestyle factors can lead to sleep disorders, which consist of disorders that can cause significant alterations in the physical, occupational, cognitive and social functioning of the individual, as well as substantially impair their quality of life $(Q \circ L)^{(6,9,10)}$. According to the recent International Classification of Sleep Disorders (ICSD), these disorders are identified in seven major categories that include insomnia disorders, sleep-related breathing disorders, central disorders of hypersomnia, sleep-wake disorders of the circadian rhythm, sleep-related movement disorders, parasomnias, and other sleep ${ }^{(11)}$.

Corresponding author Luis Felipe Tubalgi Polito. Address: Universidade Metodista de São Paulo R. Alfeu Taváres, 149 - Rudge Ramos, São Bernardo do Campo (SP), Brazil. 09641-000 E-mail: luis.polito@uol.com.br

${ }^{1}$ Escola de Ciências Médicas e da Saúde - Universidade Metodista de São Paulo, São Bernardo do Campo, SP - Brasil

2 Curso de Educação Física - Universidade de Guarulhos, Guarulhos, SP - Brasil

${ }^{3}$ Grupo de Estudos em Psicofisiologia e Treinamento Aplicados ao Exercício e ao Esporte, São Paulo, SP - Brasil

${ }^{6}$ Programa de Mestrado e Doutorado em Educação Física - Universidade São Judas, São Paulo, SP - Brasil.

Full list of author information is available at the end of the article.

Financial support: The authors declare that they did not receive funds for the study.

Submission date 20 December 2018; Acceptance date 01 February 2019; Publication date 22 March 2019 
In his epidemiological study conducted in the city of São Paulo, Castro et al. (2013) ${ }^{(12)}$ observed a high prevalence of individuals affected by symptoms of sleep disorders, which corresponded to $32 \%$ of the evaluated sample. According to these data, Silva et al. (2014) ${ }^{(13)}$ compared and described the prevalence of sleep complaints during three decades between 1987 and 2007 in the city of São Paulo. The authors observed that the percentage of individuals who experienced disturbances during sleep increased significantly, especially complaints related to difficulty sleeping, sleep at night and difficulty waking up. It is an aggravating fact that sleep disturbances coupled with an unhealthy lifestyle may contribute to a wide range of morbidities and possibly mortality ${ }^{(3,14)}$.

Therefore, the positive effect of physical exercise practice as a non-pharmacological strategy in relation to quality of life and sleep has been suggested, since it may contribute to the treatment and prevention of some disturbances of the sleep and wake cycle through reduction of sleep fragmentation, increased slow-wave sleep, and decreased latency $(4,5,10,15)$. These benefits may be relevant for sedentary individuals, since physical exercise can indirectly improve sleep quality by controlling weight and acquiring healthy habits ${ }^{(16)}$.

Among the possible mechanisms that lead to the improvement of sleep quality as a result of the exercises practice, it can be considered that the distribution of sleep stages is modified by physical exercise, since sleep is adjusted to the daily needs recovery of the organism; and, therefore, large proportions of deep sleep reflect a high recovery demand ${ }^{(17-19)}$. Additionally, during the NREM sleep stage, the ideal environment for anabolic functions, such as increased growth hormone secretion (GH), appears to be associated with slow wave sleep ${ }^{(8)}$.

However, although studies have suggested that exercise improves sleep quality ${ }^{(10,15,17,18)}$ other studies ${ }^{(20,21)}$ suggest that the effects of exercise are only modest or that they point to the fact that the effects depend on whether subjects are physically trained or not. Indeed, since the divergence between the outcomes related to the effect of physical exercise on sleep and, also, that the latter is an essential element for QoL and health, it is possible that a physical exercise program may influence the sleep quality in sedentary individuals?

Thus, it is hypothesized that sleep is the main form of reestablishment of certain functions of the organism and it seems that this can act as a response to a given stimulus, that is, the performance of physical exercises, provided that performed with moderate loads $s^{(4,8,18,21,22)}$. Additionally, as previously mentioned, physical exercise may be relevant for sedentary individuals, through weight control and acquisition of healthy habits, which may possibly contribute indirectly to a better sleep quality. Therefore, the study aims to evaluated the influence of exercise program on sleep quality of sedentary individuals.

\section{METHODS}

The study evaluated 30 beginners in resistance training subjects ( 15 male and 15 female), from August of 2018 to October of 2018. The study protocol was approved by the Ethics committee (CEP) under protocol No 59891516.6.0000.5508. The study was characterized as descriptive study, where the sleep quality was evaluated in the beginning and in the ending a specific resistance training program. Follow the characteristics of the sample: weight and height pre-program (75.49 kg and $169.85 \mathrm{~m}$, respectively) and post-program (74.55 kg and $169.86 \mathrm{~m}$, respectively). The exclusion criteria were: subjects who practiced physical activity for a period superior of three months; subjects who didn't execute all the training sessions of the program.

\section{Resistance training program}

All the sample were submitted to follow phases: 1 ) initial physical evaluation (weight and height) and application of the Pittsburgh Sleep Quality Index; 2) first workout followed by physical education professional; 3) second workout followed by physical education professional; 4) nutritional orientation; 5) final physical evaluation and second application of the PSQI. The resistance training was constituted by six multi-joints exercises (leg press, chest press, machine let pull down, seated machine shoulder press, crunches and machine trunk extension), 30 seconds rest, three times a week for 40 days. After the warm up (three minutes), the Physical Education Professional learns the correctly form of the all exercises of the workout. The intensity of the effort was controlled using the follow criteria: the ideal repetitions for each set was between eight and fifteen. This way, when the subject did sixteen repetitions with a specific load $(\mathrm{kg})$, this variable was increased to reduce the number of repetitions through the increasing of the intensity of the training. On the other hand, when the load was sufficient high to impair more of eight repetitions, this variable was reduced. In the second workout followed by physical education professional, it was evaluated the execution's technical of each exercise of the session and the training load (weight). It is important to affirm that this a real resistance training program in one of the biggest gym's net in Brazil. Besides that, the authors of the current research didn't manipulate the program, which turn to able to verify the real applicability of this study.

\section{Pittsburgh Sleep Quality Index}

The PSQI was created by Buysse et al. and evaluate the last month sleep quality, different from other questionnaires that evaluate just the last night. In its elaboration, the instrument was applied for different groups (group 1 - good sleepers; groups 2 and 3 - bad sleepers). The questionnaire consists in nineteen different questions self-determined and five questions answered for the partner room (just for clinical information). The nineteen questions are grouped in 
seven different components with scores distributed in scales from zero to three. The punctuation of the follow categories (subjective sleep quality, sleep latency, sleep duration, habitual sleep efficiency, sleep disorders, use of medicines to sleep and daytime dysfunction) are summed to result in global final score from 0 to twenty-one, where high values of the global score corresponds to bad sleep quality.

\section{Statistical analysis:}

Non-parametric effect size ( $r$ ) was calculated by taking the $z$ score and dividing it by square root of the total sample size as suggested by Rosenthal $(1994)^{(23)}$. Threshold values foe effect size statistics were $0.1,0.3,0.5$ for small, medium and large effect sizes, respectively.

\section{RESULTS}

Descriptive statistics and effect sizes for the PSQI sub-scores are presented in table 1. There was a significant effect of short-term resistance training on all sub-scores of PSQI $(p<0.05)$, except for sleep duration $(p>0.05)$. Effect sizes for the improvements in all subjective sleep indicators were considered large.

\section{DISCUSSION}

The purpose of this study was to evaluate the influence of the regular practice of physical exercise in the sleep quality among adults.

There are several processes executed during the sleep, and these processes are very important for health maintenance, including: memory consolidation, restoration of nervous system, clearance of brain metabolites etc ${ }^{(24)}$. This way, it is safe to affirm that the sleep disturbances such as sleeping time ( $<6$ hours or $>8$ hours), obstructive sleep apnea (OSA), insomnia (initiating or maintaining sleep) have a significant correlation with some metabolic diseases (diabetes, for example $)^{(25,26)}$ analysed the data from thirty-six studies and the authors concluded that the risk of developing diabetes induced by sleep disturbances is equivalent of the risk

Table 1. Slep quality parameters

\begin{tabular}{lllc}
\hline \multicolumn{1}{c}{ Parameters } & Baseline & Post & Effect size $(r)$ \\
\hline Sleep latency & $1(0-3)$ & $0(0-1)^{*}$ & -0.82 \\
Sleep duration & $2(0-3)$ & $0(0-1)^{*}$ & -0.76 \\
Habitual sleep efficiency & $0(0-3)$ & $0(0-3)^{*}$ & -0.27 \\
Sleep disturbance & $2(0-3)$ & $0(0-1)^{*}$ & -0.72 \\
Use of sleeping medications & $1(0-2)$ & $0(0-1)^{*}$ & -0.69 \\
Daytime dysfunction & $0(0-3)$ & $0(0)^{*}$ & -0.53 \\
PSQI score & $1(0-2)$ & $0(0-1)^{*}$ & -0.72 \\
\hline
\end{tabular}

Note: Values expressed in mean \pm DP. PSQI: Pittsburgh Sleep Quality Index. ${ }^{*} p<0.05$ induced by traditional risk factors (overweight, family history of diabetes etc).

So, it is necessary to discuss the variables that could contribute for reduction of the problems associated with sleep, including the non-pharmacological methods, where it is possible to include the regular practice of physical exercise ${ }^{(27)}$ Besides that, Silva et al. (2014) showed that inadequate levels of sleep duration are associated with increase of overweight and obesity in adolescents ${ }^{(28)}$.

Using the PSQI, Caldwell et al. (2009) ${ }^{(29)}$ demonstrated that "Pilates" and "Taiji" (both exercise models that include breath control and mental concentration), executed for one semester were sufficient to improve the sleep quality in college-age subjects. The same instrument was used in the study of Reid et al. (2010), and the data are in concordance with the anterior study. Reid et al. (2010) assessed seventeen sedentary adults aged $>55$ years and found that 16 weeks of aerobic physical activity plus sleep hygiene were sufficient to increase the sleep quality too ${ }^{(30)}$.

It is possible to affirm that the sleep begins parallel to the decreasing of the body temperature ${ }^{(31)}$. This way, the physical exercise would promote the increasing in body temperature, and then, would create an appropriate condition to the beginning of the sleep, since the physical exercise promotes the heat dissipation, controlled by the hypothalamus. There are two different theories that could explain the association between exercise and sleep: 1) the energy conservation and, 2) tissue restitution ${ }^{(30)}$. It is safe to affirm that the both theories have their scientific support in the homeostatic mechanisms involved in the sleep regulation, while the first one affirms that during the sleep occurs a significant reduce in metabolic rate generating a positive caloric balance. How the exercise increases the energy expenditure, the sleep is stimulated in order to generate a positive caloric balance and new vigil episode. The tissue restitution explains that the anabolic activity during the sleep is necessary after a catabolic stimulation during the vigil. Besides that, the literature is clear when affirms that the sleep provided by the exercise shows an increasing in slow-wave sleep, which is a good indicator of sleep quality ${ }^{(31,32)}$.

The study of D'Aurea et al. (2018) $)^{(33)}$ evaluated the effects of 4-month of resistance training and stretching programmes in treatment of insomnia. The authors found that both interventions are sufficient to promote significant improvement in Insomnia Severity Index scores, PSQI global scores. On the other hand, when the polysomnography or QoL measures were evaluated, no significant differences were observed. In order to evaluate the influence of different training programmes in reducing blood pressure during sleep in hypertensive elderly patients, Bertani et al. $(2018)^{(34)}$ evaluated 
sixty subjects divided in three different groups (resistance training, continuous and interval aerobic training and control). The authors find greater nocturnal decline in diastolic blood pressure compared to the wakefulness period was observed in resistance training group (after 12 weeks).

\section{CONCLUSION}

In conclusion, the resistance training program was able to improve the sleep quality in healthy subjects. In addition, the applicability of low-cost instruments (psychometric instruments like PSQI) to evaluate health parameters, which could be strengthened by other studies.

\section{Acknowledgments}

We would like to thanks the CAPES Brazilian Federal Agency for Support and Evaluation of Graduate Education within the Ministry of Education of Brazil, Methodist University of São Paulo, Guarulhos University and São Judas Tadeu University.

\section{AUTHOR'S CONTRIBUTION}

JM collected the data. HMG and MVBJ participated in the preparation of the data analysis. DPM, CGPSM and RLR wrote and read the article. LFTP, MRFB and MCZ did the final review of the paper.

\section{CONFLICTS OF INTEREST}

The author(s) declare that they have no competing interests.

\section{AUTHORS DETAILS}

4 Programa de Pós-graduação em Ciências Multidisciplinares da Saúde - Universidade Cruzeiro do Sul, SP - Brasil. ${ }^{5}$ Instituto de Ensino e Pesquisa do Hospital Israelita Albert Einstein, São Paulo, SP - Brasil. ${ }^{7}$ Curso de Educação Física- Universidade Paulista, São José do Rio Pardo - Brasil

\section{REFERENCES}

1. Foster RG, Kreitzman L. The rhythms of life: what your body clock means to you! Experimental physiology. 2014; 99(4): 599-606.

2. Alóe F, Azevedo AP, Hasan R. Sleep-wake cycle mechanisms. Revista Brasileira de Psiquiatria. 2005; 27: 33-9.

3. Kryger MH. Atlas of Clinical Sleep Medicine: Expert consult-Online and Print: Elsevier Health Sciences; 2009.

4. Martins PJF, Mello MTD, Tufik S. Exercício e sono. Revista Brasileira de Medicina do Esporte. 2001; 7(1): 28-36.

5. Montgomery P, Dennis JA. Physical exercise for sleep problems in adults aged 60+. Cochrane Database of Systematic Reviews. 2002(4).

6. Müller MR, Guimarães SS. Impacto dos transtornos do sono sobre o funcionamento diário e a qualidade de vida. Estudos de psicologia. 2007; 24(4): 519-28.

7. Roehrs T, Roth T. Sleep-wake state and memory function. Sleep. 2000; 23 : S64-8.

8. Caia, J., Kelly, V. G., \& Halson, S. L. (2018). The role of sleep in maximising performance in elite athletes. In M. Kellmann \& J. Beckmann (Eds.), Sport, recovery, and performance: Interdisciplinary insights (pp. 151-167). New York, NY, US: Routledge/Taylor \& Francis Group.

9. Geoffrey PA, Scott J, Boudebesse C, Lajnef M, Henrique C, Leboyer M, et al. Sleep in patients with remitted bipolar disorders: a meta-analysis of actigraphy studies. Acta Psychiatrica Scandinavica. 2015; 131(2): 89-99.
10. Bittencourt LRA, Silva RS, Santos RF, Pires MLN, Mello MT. Sonolência excessiva. Brazilian Journal of Psychiatry. 2005; 27: 16-21.

11. Sateia MJ. International Classification of Sleep Disorders-Third Edition. CHEST. 2014; 146(5): 1387-94.

12. Castro LS, Poyares D, Leger D, Bittencourt L, Tufik S. Objective prevalence of insomnia in the São Paulo, Brazil epidemiologic sleep study. Annals of neurology. 2013; 74(4): 537-46

13. Silva ML, Melo RJP, Dabbicco P, Freita CMSM. Sleep during, overweight and obesity in adolescence: a systematic review. Manual Therapy, Posturology \& Rehabilitation Journal. 2014; 12: 195.

14. Irwin MR, Olmstead R, Carroll JE. Sleep disturbance, sleep duration, and inflammation: a systematic review and meta-analysis of cohort studies and experimental sleep deprivation. Biological psychiatry. 2016; 80(1): 40-52.

15. Larun L, Brurberg KG, Odgaard-Jensen J, Price JR. Exercise therapy for chronic fatigue syndrome. Cochrane Database of Systematic Reviews. 2016(2)

16. Mello MT, Fernandez AC, Tufik S. Levantamento epidemiológico da prática de atividade física na cidade de São Paulo. Revista Brasileira de Medicina do Esporte. 2000; 6(4): 119-24

17. Driver HS, Taylor SR. Exercise and sleep. Sleep medicine reviews. 2000; 4(4): 387-402.

18. Knufinke M, Nieuwenhuys A, Geurts SA, Møst EI, Maase K, Moen MH, et al. Train hard, sleep well? Perceived training load, sleep quantity and sleep stage distribution in elite level athletes. Journal of science and medicine in sport. 2018; 21(4): 427-32.

19. Shapiro CM, Bortz R, Mitchell D, Bartel P, Jooste P. Slow-wave sleep: a recovery period after exercise. Science. 1981; 214(4526): 1253-4.

20. Leeder J, Glaister M, Pizzoferro K, Dawson J, Pedlar C. Sleep duration and quality in elite athletes measured using wristwatch actigraphy. Journal of sports sciences. 2012; 30(6): 541-5.

21. Youngstedt SD, Kripke DF, Elliott JA. Is sleep disturbed by vigorous late-night exercise? Medicine and science in sports and exercise. 1999; 31(6): 864-9.

22. Samuels C. Sleep, recovery, and performance: the new frontier in highperformance athletics. Neurologic clinics. 2008; 26(1): 169-80.

23. Rosenthal R, Cooper H, Hedges LV. Parametric measures of effect size. The handbook of research synthesis. 1994; 621: 231-44.

24. Luyster FS, Strollo PJ, Zee PC, Walsh JK. Sleep: a health imperative. Sleep. 2012; 35(6): 727-34.

25. Cappuccio FP, D’elia L, Strazzullo P, Miller MA. Quantity and quality of sleep and incidence of type 2 diabetes: a systematic review and meta-analysis. Diabetes care. 2010; 33(2): 414-20.

26. Anothaisintawee T, Reutrakul S, Van Cauter E, Thakkinstian A. Sleep disturbances compared to traditional risk factors for diabetes development: systematic review and meta-analysis. Sleep medicine reviews. 2016; 30: 11-24.

27. Dias RL, Oliveira LVF, Junior JRZ, Brandão GS, Silva AS, Campos FKR. Modulation of the autonomic system, pulmonary function and sleep disorders in hypertensive patients submitted to aerobic training. A study protocol. Rev. Ter. Man. 2018; 16: 594

28. Silva ML, Melo RJP, Dabbicco P, Freitas CMSM. Sleep during, overweight and obesity in adolescence: a systematic review. Manual Therapy, Posturology e Rehabilitation Journal. 2014; 12: 195.

29. Caldwell K, Harrison M, Adams M, Triplett NT. Effect of Pilates and taiji quan training on self-efficacy, sleep quality, mood, and physical performance of college students. Journal of bodywork and movement therapies. 2009; 13(2): 155-63.

30. Reid KJ, Barão KG, Lu B, Naylor E, Wolfe L, Zee PC. Aerobic exercise improves self reported sleep and quality of life in older adults with insomnia. Sleep medicine. 2010; 11(9): 934-940

31. Horne JA, Staff LHE. Exercise ond sleep: body heating effects. Sleep. 1983; 6(1): $36-46$ 
32. Horne JA, Moore VJ. Sleep EEG effects of exercise with and without additional body cooling. Electroencephalography and clinical neurophysiology. 1985; 60(1): 33-38

33. D’Aurea CV, Poyares D, Passos GS, Santana MG, Youngstedt SD, Souza $A A$, et al. Effects of resistance exercise training and stretching on chronic insomnia. Brazilian Journal of Psychiatry. 2018(AHEAD).
34. Bertani RF, Campos GO, Perseguin DM, Bonardi JM, Ferriolli E, Moriguti JC, et al. Resistance Exercise Training Is More Effective than Interval Aerobic Training in Reducing Blood Pressure During Sleep in Hypertensive Elderly Patients. The Journal of Strength \& Conditioning Research. 2018; 32(7): 2085-90. 